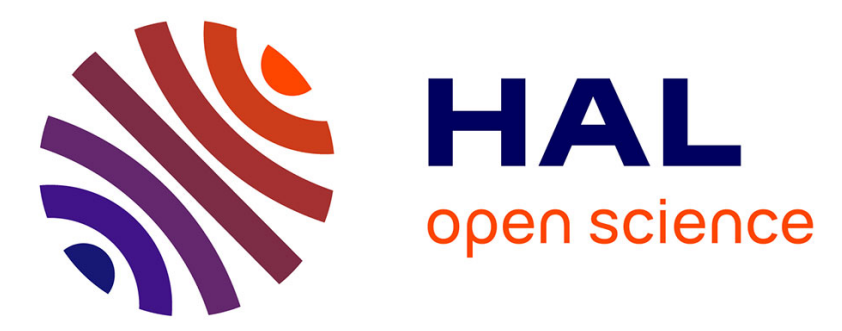

\title{
Time-Resolved Holography with Photoelectrons
}

Ymkje Huismans, Arnaud A. Rouzée, Arjan Gijsbertsen, J. H. Jungman, A. S. Smolkowska, P. S. W. M. Logman, Franck Lepine, Cécile Cauchy, Sébastien Zamith, Tatiana Marchenko, et al.

\section{- To cite this version:}

Ymkje Huismans, Arnaud A. Rouzée, Arjan Gijsbertsen, J. H. Jungman, A. S. Smolkowska, et al.. Time-Resolved Holography with Photoelectrons. Science, 2011, 331 (6013), pp.61. 10.1126/science.1198450 . hal-00553330

\section{HAL Id: hal-00553330 https://hal.science/hal-00553330}

Submitted on 7 Jan 2011

HAL is a multi-disciplinary open access archive for the deposit and dissemination of scientific research documents, whether they are published or not. The documents may come from teaching and research institutions in France or abroad, or from public or private research centers.
L'archive ouverte pluridisciplinaire HAL, est destinée au dépôt et à la diffusion de documents scientifiques de niveau recherche, publiés ou non, émanant des établissements d'enseignement et de recherche français ou étrangers, des laboratoires publics ou privés. 


\section{Time-Resolved Holography with Photoelectrons}

Y. Huismans ${ }^{1}$, A. Rouzée ${ }^{1,2}$, A. Gijsbertsen ${ }^{1}$, J.H. Jungmann ${ }^{1}$, A. S. Smolkowska ${ }^{1}$, P. S. W. M. Logman ${ }^{1}$, F. Lépine ${ }^{3}$, C. Cauchy ${ }^{3}$, S. Zamith ${ }^{4}$, T. Marchenko ${ }^{5}$, J. M. Bakker ${ }^{6}$, G. Berden ${ }^{6}$, B. Redlich ${ }^{6}$, A.F.G. van der Meer $^{6}$, H. G. Muller ${ }^{1}$, W. Vermin ${ }^{7}$, K.J. Schafer ${ }^{8}$, M. Spanner ${ }^{9}$, M. Yu. Ivanov $^{10}$, O. Smirnova ${ }^{2}$, D. Bauer ${ }^{11}$, S. V. Popruzhenko ${ }^{12}$ and M. J. J. Vrakking ${ }^{1,2}$

${ }^{1}$ FOM-Institute AMOLF, Science Park 113, 1098 XG Amsterdam, The Netherlands

${ }^{2}$ Max-Born-Institut, Max Born Straße 2A, D-12489 Berlin, Germany

${ }^{3}$ Université Lyon I, CNRS, LASIM, UMR 5579, Bât. Kastler, 43, Boulevard du 11 Novembre 1918, F69622 Villeurbanne Cedex, France.

${ }^{4}$ Laboratoire Collisions, Agrégats, Réactivité, IRSAMC, UPS, Université de Toulouse and UMR 5589 CNRS, 31062 Toulouse, France

${ }^{5}$ UPMC Université Paris 6, CNRS, UMR 7614, Laboratoire de Chimie Physique-Matière et Rayonnement, 11 rue Pierre et Marie Curie, F-75005 Paris, France 
${ }^{6}$ FOM-Institute for Plasma Physics Rijnhuizen, Edisonbaan 14, 3439 MN Nieuwegein, The Netherlands

${ }^{7}$ SARA, Science Park 121, 1098 XG Amsterdam, The Netherlands

${ }^{8}$ Department of Physics and Astronomy, Louisiana State University, Baton Rouge, Louisiana 70803-4001, USA

${ }^{9}$ National Research Council of Canada, Ottawa, Ontario K1A OR6, Canada

${ }^{10}$ Imperial College, London SW7 2BW, United Kingdom

${ }^{11}$ Institut für Physik, Universität Rostock, 18051 Rostock, Germany

12 National Research Nuclear University "Moscow Engineering Physics Institute", Kashirskoe Shosse 31, Moscow, 115409, Russia 


\begin{abstract}
Ionization is the dominant response of atoms and molecules to intense laser fields and is at the basis of several important techniques, such as the generation of attosecond pulses that allow to measure electron motion in real time. We present experiments where metastable Xenon atoms are ionized by intense $7 \mu \mathrm{m}$ laser pulses from a free electron laser. Holographic structures are observed that record underlying electron dynamics on a sub-laser cycle time scale, enabling photoelectron spectroscopy with a time resolution almost two orders of magnitude higher than the duration of the ionizing pulse.
\end{abstract}

After a strong laser field ionizes an atom or molecule, the liberated electron is accelerated by the oscillatory laser electric field and driven back towards the ion (1). Electron-ion recollision leads to the emission of XUV radiation with a duration that approaches the atomic unit of time (24.2 as) (2, 3), and encodes detailed structural and dynamical information about the atomic or molecular medium used $(4,5,6,7)$. Alternatively, the returning electron may elastically or inelastically scatter $(8,9)$. These processes benefit from the $10^{11} \mathrm{~A} / \mathrm{cm}^{2}$ electron re-collision current incident on the target ion, exceeding current densities used in transmission electron microscopes (10). The laser-driven electron motion is fully coherent, allowing to put into practice the concept of holography (11), and to extend it to electron-ion collisions involving laser-ionized and -driven 
photoelectrons $(9,12,13)$. We demonstrate how under suitably chosen experimental conditions a hologram can be recorded that encodes temporal and spatial information both about the ion (the "target") and the re-collision electron (the "source"), opening the way to a new type of ultrafast photoelectron spectroscopy of electron and nuclear dynamics in molecules.

Key to holographic electron imaging is the observation of an interference pattern between a reference wave, which is emitted from the source and does not interact with the target, and a signal wave, which scatters off the target and encodes its structure. The encoded information is stored when the signal wave interferes with the reference wave on a detector. A simple analysis borrowed from ray optics (Fig. 1A), shows that due to path length differences a phase difference $\Delta \phi=\left(\mathrm{k}-\mathrm{k}_{\mathrm{z}}\right) \mathrm{z}_{0}$ (where $\mathrm{k}$ is the total momentum, $\mathrm{k}_{\mathrm{z}}$ the momentum in the $\mathrm{z}$ direction and $\mathrm{z}_{0}$ the distance to the scattering centre) arises between the reference and scattered waves, resulting in the pattern shown in Fig. 1B.

To record a clear holographic picture it is desirable that the reference wave is not influenced by the positively charged target and, therefore, that the electron source is located at some distance from the target, $\mathrm{z}_{0}$. A suitable way to accomplish this is tunnel ionization in a strong low-frequency laser field, where the electron tunnels through a barrier created by the laser field and appears at some distance from the ion.

In the presence of the laser field the electronic wave function can be written as:

$y=/$ signat $^{\prime} y_{\text {rej }}(1)$ 
where $\psi_{\text {signal }}$ represents a signal wave packet that oscillates in the laser field and scatters off the target, and $\psi_{\text {ref }}$ represents a reference wave packet, which only experiences the laser field and does not interact with the target (14). To calculate the interference pattern produced by these two terms, we use an extension of the Strong Field Approximation (SFA), which includes the laser field fully and the electron-ion scattering in the first Born approximation $(15,16)$. The result of the calculation (Fig. S3A) (14) predicts that in a strong laser field the holographic fringes remain visible and that the phase difference between the signal and the reference wave packets is:

$\Delta \phi \approx p_{r}^{2}\left(t_{C}-t_{0}^{r e f}\right) / 2(2)$

Here $p_{r}$ is the momentum perpendicular to the laser polarization axis, $t_{C}$ the time when the signal wave packet scatters off the ion, and $t_{0}{ }^{r e f}$ the moment of birth of the reference wave packet. Thus, the hologram can be viewed as a pump-probe experiment on the fs to sub-fs timescale (Fig. S3B,C), which can encode changes in the scattering potential between $t_{0}{ }^{r e f}$ and $t_{C}$, as well as changes in the ionization rate between $t_{0}{ }^{\text {ref }}$ and $t_{0}{ }^{\text {signal }}$, the time-of-birth of the signal wave packet (14). Importantly, the signal and reference wavepackets that produce the holographic pattern originate from the same quarter cycle. Thus, sub-cycle time resolution is encoded even when long pulses are used.

A crucial aspect in our holographic imaging approach is the existence of a large electron oscillation amplitude $\alpha>>1 \AA$ and a large average oscillation energy $U_{p}>>\hbar \omega_{\text {laser }}$, where $\omega_{\text {laser }}$ is the laser frequency. In experiments with $800 \mathrm{~nm}$ radiation these requirements lead 
to high laser intensities ( $\sim 10^{14} \mathrm{~W} / \mathrm{cm}^{2}$ ), that can only be applied to ground-state atoms and molecules with a large ionization potential. To make re-collision based imaging possible at lower intensities, the laser wavelength $\lambda_{\text {laser }}$ must be increased, as both $\alpha$ and $\mathrm{U}_{\mathrm{p}}$ scale as $\lambda_{\text {laser }}^{2}$.

To demonstrate the strong field electron holography experimentally, metastable (6s) Xenon atoms were ionized by $7 \mu \mathrm{m}$ mid-infrared (mid-IR) radiation from the FELICE (Free Electron Laser for Intra-Cavity Experiments) beamline at the FELIX facility (17) (Fig. S1) (14). The use of a large $\lambda_{\text {laser }}$ in combination with a modest Ionization Potential $(I P=3.8 \mathrm{eV})$ allows the preparation of electron wave packets born at large $z_{\mathrm{o}}=\boldsymbol{I P I} \boldsymbol{F}_{\text {lase }}$, with $F_{\text {laser }}$ the laser field strength, displaying a large excursion $\alpha_{0}$, without the need for a very high laser intensity $\left(7 \times 10^{11} \mathrm{~W} / \mathrm{cm}^{2}\right)$, and remaining in the tunneling regime

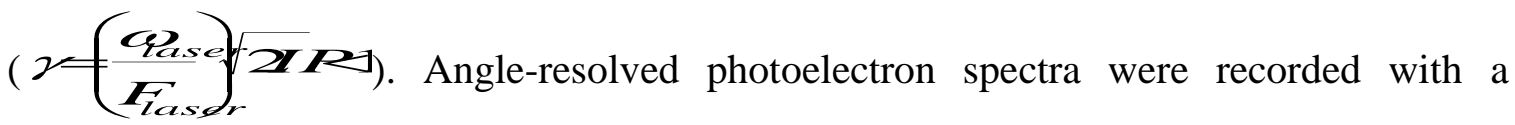
velocity map imaging spectrometer (VMI) (18) integrated into the FELICE laser cavity. The metastable Xenon atoms were exposed to a train of 5000 mid-IR laser pulses separated by $1 \mathrm{~ns}$.

Varying the position of the experimental apparatus along the laser propagation axis allowed the peak intensity to be tuned by approximately a factor 5. Fig. 2A-F show a dominant electron emission along the laser polarization axis with a high-energy cut-off (Fig. 2G) that agrees well with the classical expectation $\mathrm{E}_{\text {cut-off }}=\mathrm{F}_{\text {laser }}^{2} / 2 \omega_{\text {laser }}^{2}$. In Fig. 3A, side-lobes are observed that extend from low to high momentum and run parallel to the laser polarization axis for high momenta. These side-lobes qualitatively agree with the 
patterns calculated in Fig. S3A and result from a holographic interference. Additionally, a number of weaker transverse structures extend sideways approximately orthogonal to the laser polarization. Neither of these structures should be confused with the so-called 'sidelobes', 'wings' or 'rings' caused by backscattered electrons that were observed in higherorder Above Threshold Ionization $(19,20)$, nor are they related to the interferences observed in recent experiments on ionization of He by a few-cycle pulse (21).

The experimental observation of holographic interferences is confirmed by full TimeDependent Schrödinger Equation (TDSE) calculations, which show the same fringe pattern (Fig. 3B) (22). The fringe spacing agrees with the experiment and is reduced compared to the SFA-based calculation (Fig. S3A), where the long-range Coulomb potential was neglected.

Insight into the role of the Coulomb potential is gained by performing semi-classical calculations using the Coulomb-Corrected Strong-Field Approximation (CCSFA) (14) (23). In these calculations, complex quantum trajectories are calculated that, after tunneling, include the Coulomb interaction of the electron in the classically allowed region. The spectrum is calculated by summing contributions from different trajectories including their phases (14). The results (Fig. 3C) quantitatively reproduce the main features discussed above. Inspection of the trajectories responsible for the side-lobes shows that these trajectories can indeed be considered as a reference and scattered wave packet creating a hologram (Fig. 4A).

The efficiency of electron-ion re-collision drops dramatically with increasing $\lambda_{\text {laser }}$, due to spreading of the wave packet between ionization and re-collision. Still, a clear hologram 
can be observed at $7 \mu \mathrm{m}$. Two effects make this possible. First, the hologram results from a heterodyne experiment, where a weaker signal is mixed with a stronger signal. Second, to create a clear reference a large impact parameter is needed to limit the interaction with the Coulomb field. For large $\lambda_{\text {laser }}$ a small $p_{r}$ already leads to large impact parameters due to the long excursion time between ionization and re-collision.

Inspection of the electron trajectories contributing to the transverse structures (Fig. 3) reveals that they are due to re-collision events where the scattering does not occur on the first opportunity, but on the $2^{\text {nd }}$ or $3^{\text {rd }}$ one $(20,24,25)$. Typical examples of these trajectories are shown in Fig. 4B-D. One, resp. two glancing electron-ion collisions can be observed before the real re-collision takes place. Usually these rare events do not leave an imprint on the photoelectron spectrum. However, the combination of a long laser wavelength and Coulomb focusing (24) increases the probability, because a small deviation introduced by the Coulomb potential can be sufficient to focus the returning wave packet onto the ion.

In our model study on the ionization of metastable Xenon, we have experimentally shown the possibility to record holographic structures. Furthermore, our theoretical exploration shows that the hologram stores spatial and temporal information about the core- and electron dynamics. This offers exciting opportunities to extend strong field holography to more complicated systems and to use it to time-resolve electron-dynamics. As revealed in recent experiments $(6,26)$, electron-ion re-collision phenomena encode hole dynamics that occurs in ions during the first few femtoseconds after strong field ionization. When properly implemented with the use of a long wavelength driving laser, photo-electron 
holography appears especially well suited for studying this type of dynamics, in particular in molecules with a low binding energy that cannot easily be studied by other means. 


\section{Figure Captions}

Fig.1. (A) Diagram illustrating the concept of electron holography. Two interfering paths with the same final momentum $\mathbf{k}=\left(\mathrm{k}_{\mathrm{z}}, \mathrm{k}_{\mathrm{r}}\right)$ are indicated. Path $\mathrm{I}$ is a reference wave, leaving the source with momentum $\mathbf{k}=\left(\mathrm{k}_{\mathrm{z}}, \mathrm{k}_{\mathrm{r}}\right)$. Path II is a signal wave, incident on the target with $\mathrm{k}_{\mathrm{r}}=0, \mathrm{k}_{\mathrm{z}}=\mathrm{k}=|\mathbf{k}|$ and scattering into $\mathrm{k}=\left(\mathrm{k}_{\mathrm{z}}, \mathrm{k}_{\mathrm{r}}\right)$. The phase difference $\Delta \phi=\left(\mathrm{k}-\mathrm{k}_{\mathrm{z}}\right) \mathrm{z}_{0}$ that follows from the path length differences leads to interference fringes $\sim \cos \left[\left(k-k_{\mathrm{z}}\right) \mathrm{z}_{0}\right]$ (B) Interference pattern generated by a Gaussian wave packet released at a distance $\mathrm{z}_{0}=50$ a.u. from a scattering centre, with no laser field present. A hologram is created as a result of interference between a scattering signal wave packet and a direct reference wave packet. 
Fig. 2. Intensity dependence of measured velocity map images and the corresponding photoelectron kinetic energy distributions. (A) $7.1 \times 10^{11} \mathrm{~W} / \mathrm{cm}^{2}$; (B) $5.5 \times 10^{11} \mathrm{~W} / \mathrm{cm}^{2}$; (C) $4.5 \times 10^{11} \mathrm{~W} / \mathrm{cm}^{2}$; (D) $3.2 \times 10^{11} \mathrm{~W} / \mathrm{cm}^{2}$; (E) $2.5 \times 10^{11} \mathrm{~W} / \mathrm{cm}^{2}$ and (F) $1.9 \times 10^{11} \mathrm{~W} / \mathrm{cm}^{2} .(\mathrm{G})$ Angle-integrated photoelectron spectra. Squares indicate the energy that corresponds to the classical $2 \mathrm{U}_{\mathrm{p}}$ cut-off energy. 
Fig. 3. Comparison of an experimental 2D photo-electron velocity map image with calculations (CCSFA and TDSE) (A) 4-hour long measurement of the ionization of metastable Xenon under conditions similar to those used in the measurements shown in fig. 2A. (B) TDSE-calculation for ionization of $\operatorname{Ar}(5 \mathrm{~s})$ (IP=0.14 a.u., 4-cycle flat-top 7 $\mu \mathrm{m}$ pulse, peak field strength: 0.0045 a.u.). (C) CCSFA-Calculation for ionization of a model hydrogenic atom $\left(I P=0.14\right.$ a.u., $\lambda_{\text {laser }}=7 \mu \mathrm{m}$, peak field strength: 0.0045 a.u. $)$ Inset: CCSFA single half-cycle calculation, illustrating that the sidelobes are due to an interference between two trajectories that leave the atom within the same half-cycle. 
Fig. 4. (A) Two trajectories that lead to the formation of electrons with a final momentum $\mathrm{p}_{\mathrm{r}}=-0.01$ a.u., $\mathrm{p}_{\mathrm{z}}=-0.46$ a.u. The red trajectory corresponds to an electron that only weakly interacts with the ionic core. The blue trajectory corresponds to an electron that strongly interacts with the ionic core and that undergoes Coulomb focusing. (B-D) Recollision trajectories, illustrating the occurrence of a recollision at the first (ring 1), second (ring 3) or third (ring 2) opportunity. The highest kinetic energy of electrons on ring1 is 1.289 a.u. $\left(10.75 \mathrm{U}_{\mathrm{p}}\right)$. 


\section{References}

1. P. B. Corkum, Phys. Rev. Lett. 71, 1994-1997 (1993).

2. F. Krausz, M. Y. Ivanov, Rev. of Mod. Phys. 81, 163-234 (2009).

3. M. F. Kling, M. J. J. Vrakking, Annu. Rev. Phys. Chem. 59, 463-492 (2008).

4. J. Itatani, et al., Nature 432, 867-871 (2004).

5. D. Shafir, Y. Mairesse, D. M. Villeneuve, P. B. Corkum, N. Dudovich, Nat. Phys. 5, 412-416 (2009).

6. O. Smirnova et al., Nature 460, 972-977 (2009).

7. S. Baker et al., Science 312, 424-427 (2006)

8. M. Meckel et al., Science 320, 1478-1482 (2008).

9. M. Spanner, O. Smirnova, P. B. Corkum, M. Y. Ivanov, J. Phys. At. Mol. Opt. Phys. 37, L243-L250 (2004).

10. H. Niikura et al., Nature 417, 917-922 (2002).

11. D. Gabor, Nobel Lectures, Physics 1971-1980. World Scientific: Singapore, 1992.

12. G. G. Paulus et al., Phys. Rev. Lett. 84, 3791-3794 (2000).

13. J. Mauritsson et al., Phys. Rev. Lett. 100, 073003 (2008).

14. See supporting material on Science Online.

15. W. Becker, A. Lohr, M. Kleber, J. Phys. At. Mol. Opt. Phys. 27, L325-L332 (1994).

16. M. Lewenstein, K. C. Kulander, K. J. Schafer, P. H. Bucksbaum, Phys. Rev. A 51, 1495-1507 (1995).

17. J. M. Bakker et al., J.Chem. Phys. 132, 074305 (2010).

18. A. T. J. B. Eppink, D. H. Parker, Rev. Sci. Instrum. 68, 3477-3484 (1997). 
19. B. R. Yang et al., Phys. Rev. Lett. 71, 3770-3773 (1993).

20. G. G. Paulus, W. Becker, W. Nicklich, H. Walther, J. Phys. At. Mol. Opt. Phys. 27, L703-L708 (1994).

21. R. Gopal et al., Phys. Rev. Lett. 103, 053001 (2009).

22. H. G. Muller, Laser Physics 9, 138-148 (1999).

23. S. V. Popruzhenko, D. Bauer, J. Mod. Opt. 55, 2573-2589 (2008).

24. T. Brabec, M.Y. Ivanov, P. B. Corkum, Phys. Rev. A 54, R2551-R2554 (1996).

25. G. L. Yudin, M. Y. Ivanov, Phys. Rev. A 63, 033404 (2001).

26. Y. Mairesse et al., Phys. Rev. Lett. 104, 213601 (2010).

27. We acknowledge Rob Kemper, Hinco Schoenmaker, Ad de Snaijer, Iliya Cerjak and the staff at the FELIX facility for their great technical assistance. This work is part of the research program of the "Stichting voor Fundamenteel Onderzoek der Materie (FOM)", which is financially supported by the "Nederlandse organisatie voor Wetenschappelijk Onderzoek (NWO)". K. J. Schafer is supported by National Science Foundation grant no. PHY-0701372 and the Ball Professorship at LSU. M. Ivanov is supported by Science \& Innovation grant EP/E036112/1 of the Engineering and Physical Sciences Research Council. O. Smirnova acknowledges a Leibniz SAW award.

\section{Supporting Online Material}

WWW.sciencemag.org

Materials and Methods

SOM text 
Figs. S1, S2, S3, S4, S5

References 